\title{
The practice of nondisclosure of advanced cancer diagnosis in Singapore: a continuing challenge
}

\author{
Yung Hsiang $\underline{K a O}^{1}$, MBBS, MRCP, Cynthia Ruth $\underline{G o h}^{2}$, PhD, FRCP
}

\begin{abstract}
INTRODUCTION The traditional family-centred approach to cancer management in Singapore often leads to nondisclosure of diagnosis to patients with advanced cancer. This study aimed to determine the rate of nondisclosure to such patients in Singapore, and compare it against the rate of nondisclosure to patients' families and that of a study conducted in 1992.

METHODS Consecutive patients $(n=100)$ with advanced cancer who were referred to a palliative home care service in 2004 were studied retrospectively. Comparison between the 1992 and present study groups was performed using chisquare and Fisher's exact tests. Multivariate logistic regression was applied to patient age, Eastern Cooperative Oncology Group (ECOG) performance status, gender and ethnicity to identify factors associated with nondisclosure.

RESULTS The overall nondisclosure rate among patients with advanced cancer was 23\% (23/100), compared to only $2 \%(2 / 99)$ among their families $(p<0.001)$. The nondisclosure rates among ECOG $0-2$ and ECOG 3-4 patients were $11 \%(7 / 62)$ and $42 \%(16 / 38)$, respectively $(p<0.001)$. There was no significant improvement in the nondisclosure rate among ECOG 3-4 patients when compared to the 1992 study $(p=0.94)$. It was more likely for nondisclosure to occur among patients aged $\geq 70$ years ( $p<0.001$; odds ratio [OR] 14.77, 95\% confidence interval [CI] 3.68-59.26) and those with poor ECOG performance status ( $p=0.019$; OR 4.0, 95\% Cl 1.26-12.73). There was no significant association between nondisclosure and gender or ethnicity $(p>0.05)$.

CONCLUSION Disclosure of diagnosis to patients with advanced cancer remains a challenge in Singapore. The relationship between nondisclosure and advanced age, as well as nondisclosure and poor ECOG performance status, needs to be clarified with further studies.
\end{abstract}

Keywords: cancer, diagnosis disclosure, family-centred, palliative, Singapore

\section{INTRODUCTION}

As Singapore is a multicultural society with a heterogeneous Asian population comprising Chinese, Malays, Indians and other races, cancer management here is traditionally family-centred, which is in contrast to the patient-centred approach in Western societies. ${ }^{(1,2)}$ In the former approach, the family unit directs the patient's care and makes therapeutic and management decisions in the best interests of the patient. ${ }^{(1,2)}$ Under such social circumstances, a significant proportion of families would choose not to reveal the diagnosis to the patient. This may be due to either the family being uncomfortable with breaking the bad news or the perception that disclosure may cause depression and hopelessness, which may be misconstrued as a lack of filial piety. ${ }^{(1)}$ From a clinician's perspective, nondisclosure has serious implications, as it precludes informed consent for cancer treatment, especially enrolment in clinical trials. In 1992, Tay et $\mathrm{al}^{(3)}$ retrospectively reviewed 100 cancer patients who were under palliative home care in Singapore, and found an overall nondisclosure rate of $41 \%$ among this cohort. In another prospective study of 369 cancer patients at a Singapore radiation oncology practice in 2001, Back and Huak ${ }^{(2)}$ found an overall nondisclosure rate of $17.6 \%$.

Studies from China and South Korea have found that most patients with cancer preferred to be informed of their diagnoses..$^{(4-6)}$ Similarly, Taiwanese and Japanese studies have also reported that disclosure of diagnosis was favoured by the general population, ${ }^{(7,8)}$ and Singapore is no exception. A questionnaire survey by Chiu et $\mathrm{a}^{\left({ }^{(9)}\right.}$ in 2005 found that patients with cancer in Singapore gave the highest rating to the provision of detailed information about their medical test results. However, there is scarce data on the practice of nondisclosure among patients with cancer in Singapore. To our knowledge, only two Singapore studies have been published on this topic. ${ }^{(2,3)}$ Our study aimed to determine the nondisclosure rate among patients with advanced cancer in Singapore, and compare it to that among their families and Tay et al's(3) 1992 study. The relationship between patient factors and nondisclosure was also analysed.

\section{METHODS}

We retrospectively reviewed the medical records of a convenience sample of 102 consecutive patients referred to a palliative home care service at Assisi Hospice Home Care, Singapore, from April to September 2004. Two patients were excluded from the study due to insufficient information, leaving 100 evaluable patients. This sample size was selected to emulate Tay et al's study, which involved 100 patients. ${ }^{(3)}$ No attempt was made to calculate the sample size based on a hypothesised prevalence or to power our study's conclusions. All patients in

${ }^{1}$ Singapore Health Services, ${ }^{2}$ Department of Palliative Medicine, National Cancer Centre, Singapore

Correspondence: Dr Yung Hsiang Kao, Registrar, Singapore Health Services, 7 Hospital Drive, Block B, \#02-15, Singapore 169611. yung.h.kao@gmail.com 
our cohort had advanced cancer, which was defined as incurable, progressive locoregional or metastatic disease with limited prognosis. The home care service referred to in our study is the outpatient branch of the hospice, operated by a team of nurses and doctors who provide palliative care services to patients with advanced cancer in their homes. The primary caregiver referred to in the study was the patient's next-of-kin. Following a standard pro forma, patients and their caregivers were asked about their knowledge of the cancer diagnosis. This was done as part of the routine baseline assessment during the first home visit by a nurse or doctor.

All study data were entered into a Microsoft Excel database (Microsoft Corp, Redmond, WA, USA), with patient age presented as mean, median, range and 95\% confidence interval $(\mathrm{CI})$. Comparison against the study by Tay et $\mathrm{al}^{(3)}$ was performed using chi-square and Fisher's exact tests, where $\mathrm{p}<0.05$ was considered statistically significant. However, in order for this comparison to be statistically feasible, it was necessary to assume Tay et al's entire study population to be classified under Eastern Cooperative Oncology Group (ECOG) performance status 3-4. This assumption was essential, as Tay et al merely reported that the majority (93\%) of their study population had an ECOG 3-4 status, but did not group the patients according to their ECOG performance status. ${ }^{(3)}$ Therefore, the nondisclosure rate of ECOG 0-2 patients in our study and in Tay et al's study could not be compared. Multivariate logistic regression was applied to age, gender, ethnicity and ECOG performance status in order to identify factors related to nondisclosure.

\section{RESULTS}

Our cohort $(n=100)$ consisted of Chinese $(n=85)$, Malays $(n=5)$, Indians $(n=8)$ and patients of other ethnicities $(n=2)$. The types of cancer detected in our cohort were classified as: colorectal $(n=20)$; lung $(n=18)$; stomach $(n=11)$; liver $(n=7)$; and others $(n=44)$. No statistically significant difference between our data and published national data, ${ }^{(10)}$ with regard to patient or disease demographics (chi-square test; $p>0.05$ ), was found. Table I presents the comparison of data between our study and Tay et al's study. ${ }^{(3)}$ Although chi-square test showed no statistically significant difference in gender, age or ethnicity ( $p>0.05)$, it revealed a statistically significant difference in ECOG performance status between the two study populations $(\mathrm{p}<0.001)$.

The overall rate of nondisclosure of cancer diagnosis in our study was $23 \%$ (23 patients in our study were unaware of their diagnosis). The rates of nondisclosure in ECOG 0-2 and ECOG $3-4$ patients were $11 \%$ (7/62) and $42 \%$ (16/38), respectively, with statistically significant difference between the two (chi-square test; $p<0.001$ ). Chi-square test, however, showed no significant difference between the nondisclosure rate of ECOG 3-4 patients in our study and that in Tay et al's study ${ }^{(3)}$ ( $p=0.94)$. In our study, $2(2 \%)$ families (2 out of 99 , as one family was excluded from analysis due to incomplete data) were
Table I. Comparison of present study conducted in 2004 against Tay et al's 1992 study. ${ }^{(3)}$

\begin{tabular}{|c|c|c|}
\hline Parameter & $\begin{array}{l}\text { Tay et } \mathrm{al}^{(3)} \\
(\mathrm{n}=100)\end{array}$ & $\begin{array}{c}\text { Present study } \\
(n=100)\end{array}$ \\
\hline \multicolumn{3}{|l|}{ Gender } \\
\hline Male & 48 & 57 \\
\hline Female & 52 & 43 \\
\hline \multicolumn{3}{|l|}{ Mean age (yrs) } \\
\hline Male & 67 & 67 \\
\hline Female & 58 & 61 \\
\hline \multicolumn{3}{|c|}{ Median age (yrs) } \\
\hline Male & - & 69 \\
\hline Female & - & 58 \\
\hline \multicolumn{3}{|l|}{ Age range (yrs) } \\
\hline Male & $29-93$ & $37-91$ \\
\hline Female & $4-87$ & $9-90$ \\
\hline \multicolumn{3}{|c|}{ 95\% Cl (age; yrs) } \\
\hline Male & - & $64-71$ \\
\hline Female & - & $55-67$ \\
\hline \multicolumn{3}{|l|}{ Ethnicity } \\
\hline Chinese & 86 & 85 \\
\hline Non-Chinese & 14 & 15 \\
\hline \multicolumn{3}{|c|}{$\begin{array}{l}\text { ECOG performance } \\
\text { status }\end{array}$} \\
\hline $0-2$ & 7 & 62 \\
\hline $3-4$ & 93 & 38 \\
\hline
\end{tabular}

Note: Data is presented as number of patients unless stated otherwise. $\mathrm{Cl}$ : confidence interval; ECOG: Eastern Cooperative Oncology Group

Table II. Multivariate logistic regression analysis of patient factors and nondisclosure of cancer diagnosis.

\begin{tabular}{lrcc}
\hline Variable & OR & $95 \% \mathbf{C l}$ & p-value \\
\hline $\begin{array}{l}\text { Age (yrs) } \\
\quad<70\end{array}$ & 1.00 & $3.68-59.26$ & $<0.0001$ \\
$\quad \geq 70$ & 14.77 & & \\
Gender & & $0.79-8.55$ & 0.114 \\
$\quad$ Male & 1.00 & & \\
$\quad$ Female & 2.61 & & 0.652 \\
Ethnicity & & $0.28-7.78$ & \\
$\quad$ Chinese & 1.00 & & 0.019 \\
$\quad$ Non-Chinese & 1.46 & & \\
ECOG performance status & & $1.26-12.73$ & \\
$\quad$ 0-2 & 1.00 & & \\
3-4 & 4.00 & & \\
\hline
\end{tabular}

$\mathrm{Cl}$ : confidence interval; ECOG: Eastern Cooperative Oncology Group; OR: odds ratio

unaware of the patients' cancer diagnoses. Fisher's exact test revealed a highly statistically significant difference in knowledge of the diagnoses between patients and their families $(p<0.001)$. Multivariate logistic regression analysis showed that advanced age and ECOG performance status were significantly related to nondisclosure. Patients aged $\geq 70$ years were much more likely to be unaware of their diagnoses than those aged $<70$ years ( $p<0.001$; odds ratio [OR] 14.77, 95\% Cl 3.7-59.3). Similarly, ECOG 3-4 patients were more likely to be unaware of their diagnoses ( $p=0.019$; OR 4.0,95\% Cl 1.3-12.7) than ECOG $0-2$ patients. Both gender $(\mathrm{p}=0.114$; OR $2.61,95 \% \mathrm{Cl} 0.8-8.6)$ and ethnicity ( $p=0.652$; OR $1.46,95 \% \mathrm{Cl} 0.3-7.8$ ) showed no statistically significant relationship with nondisclosure. The results of the multivariate logistic regression analysis are summarised in Table II. 


\section{DISCUSSION}

In Singapore, the traditional family typically adopts a familycentred approach in the face of challenges, such as the disclosure of advanced cancer. ${ }^{(1,2)}$ In such a family-centred model, a patient with cancer may choose to defer medical decisions to his/her family. The family in turn makes decisions in the best interests of the patient. ${ }^{(1,2)}$ In this social context, we found a $23 \%$ overall rate of nondisclosure among patients with advanced cancer in Singapore in 2004, which was an improvement from the rate of $41 \%$ reported in 1992.(3) This finding is similar to another local study in 2001, which reported a nondisclosure rate of $17.6 \%$ among radiation oncology patients. ${ }^{(2)}$ Our findings are also supported by Chan et al's findings that $23 \%-30 \%$ of surveyed general practitioners and specialists in Singapore preferred to withhold from their patients the truth about their illnesses. ${ }^{(11)}$ The rate of nondisclosure among patients with cancer in Singapore is similar to that in other East Asian countries. A review of the literature published in the last decade showed the following nondisclosure rates in East Asia, classified according to country: Taiwan: 21\%; ${ }^{(12)}$ Thailand: 37\%; ${ }^{(13)}$ Japan: 29\%;(14) and Korea: 42\%. ${ }^{(4)}$ However, this practice of nondisclosure is in contrast to the attitude of the general East Asian population. Taiwanese and Japanese studies have found that nondisclosure was preferred by only $2.9 \%-13.9 \%$ of the general population. ${ }^{(7,8,15)}$ Studies in China, Taiwan, Japan and Korea have also found that patients with cancer have an overall preference for disclosure of diagnosis. ${ }^{(5,6,16)}$ Similarly, a questionnaire survey by Chiu et al in 2005 on 200 patients with cancer in Singapore found that the highest rating was given to doctors who provided "detailed information about the results of medical tests".(9)

Our study yielded several interesting findings. Firstly, we found a highly significant difference between patients' and their families' knowledge of the diagnosis. Secondly, analysis by ECOG performance status showed no significant improvement in nondisclosure rates among ECOG 3-4 patients from 1992 to 2004. Thirdly, multivariate logistic regression analysis found that patients with advanced cancer, those aged $\geq 70$ years and those with poor ECOG performance status (i.e. ECOG 3-4) were more likely to be uninformed of their diagnoses than patients below 70 years of age or with better ECOG performance status (i.e. ECOG 0-2). In Singapore, it is common practice for a doctor to first disclose the diagnosis of advanced cancer to a patient's family, after which the doctor will ask the family whether they would like the diagnosis to be disclosed to the patient. In 1993, Tan et al found that $84 \%$ of doctors in Singapore would accede to the family's request not to reveal the cancer diagnosis to the patient. ${ }^{(17)}$ Our study found that while only $2 \%$ of families were unaware of the patients' diagnosis, there was nondisclosure of diagnosis to $23 \%$ of patients in our cohort. This difference is highly significant, highlighting a strong possibility that the traditional Asian family-centred approach to cancer management continues to have a negative impact on disclosure of diagnosis in Singapore. Other East Asian studies have suggested that a family-centred approach may significantly and negatively impact nondisclosure of cancer diagnosis. Phungrassami et al found that Thai patients with cancer who were aware of their diagnoses were either unaccompanied by their family members, or their families were in support of disclosure of diagnosis. ${ }^{(13)}$ Jiang et al found that the families of patients with cancer in China were more likely to favour nondisclosure than the patients themselves. ${ }^{(5)}$ Yun et al also reported similar findings among Korean patients with cancer and their families. ${ }^{(4,6)}$

We found that patients with cancer who are of advanced age were more likely to be unaware of their diagnoses than younger patients. Other studies from Singapore, Thailand and Taiwan

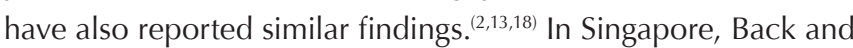
Huak reported that being of advanced age and non-Englishspeaking were strong predictors of nondisclosure among radiation oncology patients. ${ }^{(2)}$ They further suggested that among such patients, these two factors may be associated with a lower educational level. ${ }^{(2)}$ We speculate that in the traditional Singaporean family unit, such patients would usually allow younger, better educated or English-speaking family members to make medical decisions on their behalf. This would predispose nondisclosure, as younger family members may be fearful or unsure of communicating bad news, or perceive withholding bad news as an act of filial piety.

We also found that there was a higher rate of nondisclosure among patients with advanced cancer and poor ECOG performance status, which is in keeping with the findings of Back and Huak, (2) who reported a significant association between nondisclosure and palliative treatment aim. Additionally, we observed a lack of improvement in disclosure rate from 1992 to 2004 among patients with advanced cancer and poor ECOG performance status. The underlying reasons are unclear and there is a lack of literature on this issue. We speculate that this lack of improvement may be related to patients' poor prognosis. As a patient with advanced cancer and ECOG performance status $3-4$ is frequently frail and usually terminally ill, it is not uncommon for the family unit, especially in the Singaporean context of family-centric cancer management, to assume a protective role so as to shield the patient from the perceived negative impact of bad news, which is highly regarded as an act of filial piety in Singapore. ${ }^{(1)}$ Further studies are needed to expound on the relationship between nondisclosure and factors such as advanced age and poor ECOG performance status, and to explore Singaporean families' attitudes toward disclosure of cancer diagnosis to patients.

Many families in Singapore feel uncomfortable about cancer disclosure for fear that it will cause the patient to experience anxiety, develop depression or lose hope and the will to live. ${ }^{(1,2,17)}$ However, evidence from East Asia contradicts this perception. Studies have found that Taiwanese patients with cancer who were aware of their diagnosis had higher levels of hope, internal health locus of control and lower pain levels. ${ }^{(12,18)}$ Similarly, Horikawa et al reported that Japanese patients with cancer 
who were aware of their diagnosis experienced less depression and anxiety. ${ }^{(19)}$ In Korea, Yun et $\mathrm{al}^{\left({ }^{(4)}\right.}$ found that $28.5 \%$ of patients with terminal cancer who were aware of their diagnosis had already suspected it from their worsening health - this suggests that nondisclosure did not prevent patients with cancer from eventually guessing the diagnosis. The authors further argued that most of the initial negative emotional impact experienced by patients with cancer following disclosure is consistent with the Kübler-Ross grief model, and is therefore transient, appropriate and healthy. ${ }^{(4)}$ Other authors likewise feel that patients with cancer who are aware of their diagnosis will be better able to take action to resolve unfinished personal matters, engage effectively with doctors in making appropriate therapeutic decisions, experience more satisfaction with their medical care, and in some situations, even avoid futile therapy. ${ }^{(1,4,6)}$ Also, as informed consent is impossible without disclosure of diagnosis to patients, withholding the diagnosis effectively excludes patients from most, if not all, clinical trials and emerging treatment modalities.

In modern medicine, the way in which a cancer diagnosis is disclosed is more important than the decision on whether or not to do so. ${ }^{(6)}$ Thus, one of the greatest challenges in Singapore is the integration of routine disclosure into the current traditional family-centric approach to cancer management. In Western societies, disclosure of cancer diagnosis has been part of the standard of care for many years. ${ }^{(20)}$ Moreover, Western-based training materials that guide doctors on the art of cancer diagnosis disclosure are readily available. ${ }^{(21-23)}$ Unfortunately, a family-centric guideline on cancer disclosure has yet to be established in the Asian context.

A fortuitous finding in our study was the significant improvement in referral pattern for palliative home care in Singapore from 1992 to 2004. We also found a significant increase in the proportion of patients with good ECOG performance status being referred to palliative home care services $(7 \%$ in $1992^{(3)}$ vs. $62 \%$ in 2004). This means that more patients with advanced cancer in Singapore are now receiving palliative care at an earlier stage of their illness. This improvement has largely been the result of continual national commitment to improve palliative care service in the country. As a testament to these recent achievements, a multinational study ${ }^{(24)}$ on overall 'quality of death' published in 2010 ranked Singapore second (after Taiwan) in Asia and 18th globally.

In conclusion, despite the many benefits to patient care, the practice of disclosure of cancer diagnosis remains a challenge in Singapore. Although this study has identified advanced age and poor ECOG performance status as factors associated with nondisclosure, the underlying reasons are still unclear and should be investigated by further studies. As Singapore continues its socioeconomic progress as a modern, prosperous and highly educated city-state, the apparent lack of improvement in families' attitudes toward disclosure is thus of concern. We propose that disclosure of diagnosis be considered as an essential component of cancer management.

\section{ACKNOWLEDGEMENTS}

We would like to thank Ms Gao Fei (Biostatistician, Division of Clinical Trials and Epidemiological Sciences, National Cancer Centre Singapore) for her assistance in statistical analysis, and Dr Jan Hallstrom (former Medical Director, Assisi Home and Hospice, Singapore) for his encouragement and advice during the course of the study.

\section{REFERENCES}

1. Lee A, Wu HY. Diagnosis disclosure in cancer patients-when the family says "no!". Singapore Med J 2002; 43:533-8.

2. Back MF, Huak CY. Family centred decision making and non-disclosure of diagnosis in a South East Asian oncology practice. Psychooncology 2005; 14:1052-9.

3. Tay WK, Shaw RJ, Goh CR. A survey of symptoms in hospice patients in Singapore. Ann Acad Med Singapore 1994; 23:191-6.

4. Yun YH, Kwon YC, Lee MK, et al. Experiences and attitudes of patients with terminal cancer and their family caregivers toward the disclosure of terminal illness. J Clin Oncol 2010; 28:1950-7.

5. Jiang Y, Liu C, Li JY, et al. Different attitudes of Chinese patients and their families toward truth telling of different stages of cancer. Psychooncology 2007; 16:928-36.

6. Yun YH, Lee CG, Kim SY, et al. The attitudes of cancer patients and their families toward the disclosure of terminal illness. J Clin Oncol 2004; 22:307-14.

7. Wang SY, Chen $\mathrm{CH}$, Chen YS, Huang HL. The attitude toward truth telling of cancer in Taiwan. J Psychosom Res 2004; 57:53-8.

8. Miyata H, Takahashi M, Saito T, et al. Disclosure preferences regarding cancer diagnosis and prognosis: to tell or not to tell? J Med Ethics 2005; 31:447-51.

9. Chiu LQ, Lee WS, Gao F, et al. Cancer patients' preferences for communication of unfavourable news: an Asian perspective. Support Care Cancer 2006; 14:818-24.

10. Seow A, Koh WP, Chia KS, et al. Trends in cancer incidence in Singapore 1968-2002. Singapore Cancer Registry Report No. 6. Singapore: National Registry of Diseases Office, 2004.

11. Chan D, Goh LG. The doctor-patient relationship: a survey of attitudes and practices of doctors in Singapore. Bioethics 2000; 14:58-76.

12. Lin CC, Tsay HF. Relationships among perceived diagnostic disclosure, health locus of control, and levels of hope in Taiwanese cancer patients. Psychooncology 2005; 14:376-85.

13. Phungrassami T, Sriplung H, Roka A, et al. Disclosure of a cancer diagnosis in Thai patients treated with radiotherapy. Soc Sci Med 2003; 57:1675-82.

14. Horikawa N, Yamazaki T, Sagawa M, Nagata T. Changes in disclosure of information to cancer patients in a general hospital in Japan. Gen Hosp Psychiatry 2000; 22:37-42

15. Miyata H, Tachimori H, Takahashi M, Saito T, Kai I. Disclosure of cancer diagnosis and prognosis: a survey of the general public's attitudes toward doctors and family holding discretionary powers. BMC Med Ethics 2004; 5:E7.

16. Fujimori M, Uchitomi Y. Preferences of cancer patients regarding communication of bad news: a systematic literature review. Jpn J Clin Oncol 2009; 39:201-16.

17. Tan TK, Teo FC, Wong K, Lim HL. Cancer: to tell or not to tell? Singapore Med J 1993; 34:202-3.

18. Lin CC. Disclosure of the cancer diagnosis as it relates to the quality of pain management among patients with cancer pain in Taiwan. J Pain Symptom Manage 1999; 18:331-7.

19. Horikawa N, Yamazaki T, Sagawa M, Nagata T. The disclosure of information to cancer patients and its relationship to their mental state in a consultation-liaison psychiatry setting in Japan. Gen Hosp Psychiatry 1999; 21:368-73.

20. Buckman R. Talking to patients about cancer. BMJ 1996; 313:699-700.

21. Girgis A, Sanson-Fisher RW. Breaking bad news: consensus guidelines for medical practitioners. J Clin Oncol 1995; 13:2449-56.

22. Ngo-Metzger Q, August KJ, Srinivasan M, Liao S, Meyskens FL Jr. End-of-Life care: guidelines for patient-centered communication. Am Fam Physician 2008; 77:167-74.

23. Clayton JM, Hancock KM, Butow PN, et al. Clinical practice guidelines for communicating prognosis and end-of-life issues with adults in the advanced stages of a life-limiting illness, and their caregivers. Med J Aust 2007; 186(12 Suppl):S77, S79, S83-108

24. Economist Intelligence Unit. The quality of death. Ranking end-of-life care across the world. London: Economist Intelligence Unit, 2010. 